\title{
EXEMPLE EFFECTIF D'UN ENSEMBLE TRANSFINIMENT NON-PROJEGTIF
}

\author{
F. ROTHBERGER
}

1. Introduction.

2. Méthode de Lebesgue (élimination de nombres transfinis).

3. Définition effective de $\mathrm{P}_{\alpha}$ et d'ensemble non-projectif.

4. Démonstration.

5. Simplifications.

1. Introduction. La notion d'ensemble projectif ${ }^{1}$ de classe finie $P_{n}$ peut être généralisée pour les classes transfinies $P_{\alpha}(\alpha<\Omega)$. Nous allons ici construire effectivement un ensemble qui n'est pas projectif, même dans ce sens généralisé. ${ }^{2}$

Dans ce qui suit, les variables, $x, y, z$ parcourent les nombres irrationnels. Quoique pas nécessaire, cela simplifie les définitions, puisque la courbe de Peano établit une homéomorphie entre l'ensemble $J$ des nombres irrationnels et l'ensemble $J^{2}$ du plan, ou $J^{3}$ (points irrationnels de l'espace) etc. Donc, puisque les classes projectives sont topologiques, ceci nous permet, d'une sorte, de négliger le nombre de dimensions de l'espace.

On définit les ensembles analytiques $A$ comme projections des $G_{\delta}$, ou, plus simplement, comme projections des ensembles relativement fermés dans $J^{n}$. Soit $A=P_{1}$. Ensuite, on a les compléments analytiques $C A$, leurs projections $P C A=P_{2}, P C P C A=P_{3}, \ldots$

Un ensemble qui est somme (dénombrable) d'ensembles $P_{n}$ ( $n$ variable) sera de classe $P_{\omega}$, et on définira $P_{\omega+1}=P C P_{\omega}$, et ainsi de suite, pour tous les $\alpha<\Omega$. Comme on sait, la classe $A$ contient tous les ensembles $B$ de Borel; la classe $P_{2}=P C A$ contient tous les ensembles $B, A, C A$; et en général la classe $P_{\alpha}$ (pourvu qu'elle existe) contiendra toutes les classes précédentes, c'est à dire, $P_{\beta}$ et $C P_{\beta}$ pour tout $\beta<\alpha$.

On vérifie que toutes ces classes sont topologiques.

Remarque. Puisque l'opération $P$ fait perdre une dimension, on peut remplacer $P$ par $P \pi$ où $\pi$ est la transformation péanienne de la droite $J$ sur le plan $J^{2}$. C'est à dire, on transforme un ensemble linéaire en un ensemble plan, et fait la projection ensuite. On pourra aussi remplacer $P C P$ par $P C P \pi_{3}$, où $\pi_{3}$ est la transformation péanienne à trois dimensions. Ainsi on restera toujours dans la même dimension.

Reçu le 3 avril, 1957.

${ }^{1}$ Pour renseignements généraux, on pourrait consulter les livres classiques $(\mathbf{1} ; \mathbf{3})$. En particulier, pour la méthode de Lebesgue, voir (3, pp. 298-320).

${ }^{2}$ En principe, la possibilité de construction effective d'un ensemble non-projectif est connue depuis longtemps (5). Du point de vue de projectivité finie, $\mathrm{P}_{\omega}$ est déjà non-projectif $(4$, p. 12). 
Définition. Un ensemble universel pour la classe $P_{\alpha}$ est un ensemble dans l'espace $J^{n}$, ou bien, pour fixer les idées: dans le plan $(x, y)$, tel que, pour $y$ variable, les sections parallèles à l'axe des $x$ parcourent tous les ensembles linéaires de classe $P_{\alpha}$ (les classes inférieures inclus); les répétitions sont permises.

Nous allons stipuler encore que l'ensemble universel doit être, lui-même, un ensemble projectif (à plusieurs dimensions), quoique peut être d'une classe supérieure à $\alpha$.

Pour démontrer l'existence d'un ensemble proprement de classe $P_{\alpha+1}$ il suffit de démontrer l'existence d'un ensemble universel pour la classe $P_{\alpha}$. (Pour les classes limites, par exemple $P_{\omega}$, il n'y a pas de difficulté.)

Ainsi, la même méthode qui sert à démontrer que les classes $P_{1}, P_{2}, \ldots$ $P_{n}, \ldots$ sont distinctes, peut être utilisée pour les classes $P_{\alpha}$; ce qui entrainera l'existence d'ensembles proprement $P_{\alpha}$ pour tout $\alpha<\Omega$.

Notre but est de construire des ensembles projectifs universels $P_{\alpha}$ pour une suite transfinie de $\alpha \rightarrow \Omega$. Plus précisément, nous allons construire un ensemble dans l'espace $(x, y, t)$, dont, pour $t$ variable $(0 \leqslant t<1)$, les sections parallèles parcourent des ensembles universels pour des classes aussi hautes qu'on veut. Cet ensemble sera ainsi, en certain sens, universel pour tous les ensembles linéaires projectifs de toutes les classe $\alpha<\Omega$, donc, lui-même, non projectif. La définition sera formalisable en termes logiques et arithmétiques, c'est à dire, avec les symboles $\vee, \&, \rightarrow$, non, $(\exists m),(n)$ (quantificateurs du $1^{\mathrm{er}}$ ordre), $(\exists x),(y)$ (quantificateurs sur variables réelles, $2^{\text {ième }}$ ordre), et les symboles d'arithmétique; et encore un quantificateur du troisième ordre, qui intervient pour éviter une définition implicite.

Ici, les variables parcourant les nombres naturels (ou, si l'on veut, les nombres rationnels) sont considérés comme variables du $1^{\mathrm{er}}$ ordre, les variables $x, y, \ldots$, parcourant le système des nombres réels (ou bien, ce qui revient au même, parcourant le système des ensembles de nombres naturels) seront des variables du $2^{\text {ième }}$ ordre; enfin, une variable $\phi(x)$ ou $\phi(x y), \ldots$, ou encore, une variable parcourant le système de tous les ensembles de nombres réels (c'est à dire, les ensembles d'ensembles de nombres naturels) sera du troisième ordre. (Une variable irrationnelle sera du $2^{\text {ième }}$ ordre comme une variable réelle, etc.)

Remarquons que ce troisième ordre (ou bien l'implicité) est essentielle: (2); tout ensemble qui est explicitement arithmétisable avec le calcul fonctionnel du second ordre est au plus projectif de classe finie.

2. Méthode de Lebesgue pour éliminer les nombres transfinis. D'abord, soit $t$ un paramètre réel $0 \leqslant t<1$, en développement dyadique:

$$
t=0 . a_{1} a_{2} a_{3} \ldots a_{n} \ldots
$$

et soit:

$$
r_{1}, r_{2}, r_{3}, \ldots, r_{n}, \ldots
$$


une suite bien définie de tous les nombres rationnels, fixée pour tout ce qui suit.

A chaque $t$ correspond l'ensemble $T$ de tous les $r_{n}$ tels que $a_{n}=1$. Par exemple:

$$
\begin{array}{cccccccccccc}
t= & 0 \cdot & 0 & 1 & 1 & 0 & 1 & 0 & 1 & 1 & 10 & \ldots \\
T= & (\cdot, & r_{2}, & r_{3}, & \cdot, & r_{5}, & \cdot, & r_{7}, & r_{8}, & r_{9}, & \cdot, & \ldots
\end{array}
$$

Dans le cas d'ambiguité de certains $t$ rationnels convenons que $T$ soit l'ensemble fini. (L'autre, n'étant pas bien-ordonné, n'aura pas d'intérêt.) Pour définition formelle, voir définition 2 , plus bas.

Définition 1 Nous dirons que $t$ est bien-ordonné $d u$ type $\alpha$, si $T$, ordonné selon la relation <, est bien-ordonné du type $\alpha$, formellement; ord $t=\alpha$.

Définition 2 Nous écrirons $r \in t$ au lieu de $r \in T$. Ou bien, formalisé:

$$
r_{n} \in t \cdot \equiv \cdot\left[2^{n} t\right]=1(\bmod 2) .
$$

Définition 3 Soit $t_{1} \prec t_{2}$ si $T_{1}$ est un propre segment (Abschnitt) de $T_{2}$. Formellement:

$$
t_{1} \prec t_{2}: \equiv:(\exists r)\left(r^{\prime}\right) \cdot r^{\prime} \in t_{1} \cdot \equiv \cdot r \in t_{2} \& r^{\prime} \in t_{2} \& r^{\prime}<r .
$$

(on pourrait éliminer le $\in$ selon la définition 2.)

On voit sans peine que:

LEMME.

$$
\operatorname{ord} t=\left\{\begin{array}{cl}
(\max \operatorname{ord} \tau)+1, & \tau<t \\
\lim \operatorname{ord} \tau, & \tau<t
\end{array}\right\} \text { (selon les deux cas). }
$$

Ainsi, au lieu de définir des ensembles $\mathbf{E}_{\alpha}$ par induction sur $\alpha$, nous pouvons définir des ensembles $\mathbf{E}_{t}$ avec une induction sur $t$ par la relation $\prec$.

3. Définition effective des $P_{\alpha}(\alpha<\Omega)$ et d'un ensemble non-projectif. Soit $x_{1}=\pi_{3}\left(x, z, z^{\prime}\right)$ une homéomorphie entre $J$ et $J^{3}$ (courbe de Peano). Nous la supposons effectivement déterminée par une définition arithmétisée. De même, supposons donnée effectivement une autre, à une infinité de dimensions, qui fait correspondre les nombres irrationnels $y$ aux suites $\left(y_{1}, y_{2}, y_{3}, \ldots\right.$ $\left.y_{n}, \ldots\right)$ de nombres irrationnels, d'une manière biunivoque et bicontinue. $\mathrm{Au}$ lieu de la notation $y_{n}=\pi_{n}(y), n=1,2,3, \ldots$, nous écrirons

$$
y_{n}=\pi_{r_{n}}(y)
$$

(c'est à dire, remplacant l'indice naturel par un indice rationnel, qui parcourt la suite $r_{1}, r_{2}, r_{3}, \ldots$, ). Ici encore, les définitions sont arithmétisables, dans le même sens.

Maintenant nous allons définir les ensembles annoncés dans l'introduction par une fonction propositionnelle $\phi(t, x, y),(0 \leqslant t<1, x \in J, y \in J)$. Ici $x$ sera la variable principale, $y$ et $t$ des paramètres. Moyennant les symboles 
logiques et arithmétiques (avec quantificateurs du $1^{\text {er }}$ et $2^{\text {ième }}$ ordre) et des notions formalisables en ce sens, cette fonction $\phi$ est définie implicitement par induction transfinie rel. $\prec$, comme il suit:

$$
\phi(0, x . y) \cdot \equiv \cdot(\exists r) \pi_{r}(y)<x<r .
$$

et, si $t$ est bien-ordonné et $t \neq 0$ :

$$
\phi(t, x, y) \cdot \equiv \cdot(\exists z)\left(z^{\prime}\right)(\exists r) \phi\left(t_{r}, \pi_{3}\left(x, z, z^{\prime}\right), \pi_{r}(y)\right)
$$

où $t_{r}$ est le segment de $t$ jusqu'à $r(r \in t)$; plus précisément,

$$
r^{\prime} \in t_{r} \equiv \cdot r \in t \& r^{\prime} \in t \& r^{\prime}<r .
$$

Ainsi, si $r \notin t$, on aura $t_{r}=0$ (c'est à dire, l'ensemble vide).

Donc, supposé que $\phi(\tau, x, y)$ est défini pour tous les $\tau \prec t(t$ bien-ordonné), la formule (2) définit $\phi(t, x, y)$. Ainsi $\phi$ est défini pour tous les $t$ bien-ordonnés.

Si $t$ n'est pas bien-ordonné, on peut définir, soit:

$$
\phi(t, x, y) \cdot \equiv \cdot 0=0, \text { (c'est à dire, toujours vrai), }
$$

soit:

$$
\phi(t, x, y) \cdot \equiv \cdot 0=1 \text {, (c'est à dire, toujours faux). }
$$

Notons que " $t$ n'est pas bien-ordonné" est formalisable ainsi:

$$
(\exists \tau)(r)\left(\exists r^{\prime}\right) r \in \tau \rightarrow r \in t . \& . r^{\prime} \in \tau \& r^{\prime}<r .
$$

Remarquons, en passant, que le quantificateur réel $(\exists \tau)$ est essentiel: en effet, c'est l'exemple classique d'un ensemble analytique; mieux arithmétisé:

$$
\begin{aligned}
& \hat{t}(\exists \tau)(m)(\exists n) \therefore r_{n}<r_{m} . \& .\left[2^{n} \tau\right]=1(\bmod 2): \& \\
& \&:\left[2^{m} \tau\right]=0 \vee\left[2^{m} t\right]=1(\bmod 2) .
\end{aligned}
$$

On pourrait combiner (2) et (3) en une formule pour tous les $t \neq 0$

$$
\phi(t, x, y): \equiv: \text { (second membre de }(2)) \cdot \vee \cdot(\text { formule }(4)) .
$$

Je dis que l'ensemble $\hat{t}, \hat{x}, \hat{y} \phi(t, x, y)$ est non-projectif.

4. Démonstration. Nous allons démontrer que ces ensembles ont les propriétés envisagées, c'est à dire, que $\hat{x} \phi(t, x, y)$ parcourt tous les ensembles projectifs et que $\hat{x} \hat{y} \phi(t, x, y)$ sont des ensembles projectifs universels.

Pour voir la signification de (2), soit

$$
E_{t, y}=\hat{x} \phi(t, x, y), \mathbf{E}_{t, y}^{3}=\hat{x} \phi\left(t, \pi_{3}\left(x, z, z^{\prime}\right), y\right), \mathbf{E}_{t}^{3}=\hat{x} \hat{y} \phi\left(t, \pi_{3}\left(x, z, z^{\prime}\right), y\right) .
$$

On a

$$
E_{t, v}=P C P C \sum_{r} E_{t_{r} \pi_{r}(y)}^{3}
$$

en vertu de $(2)$, où 


$$
\sum_{r} \boldsymbol{E}_{t_{r} \pi_{r}(y)}^{3}=\boldsymbol{E}_{t_{r_{1} y_{1}}}^{3}+\boldsymbol{E}_{t_{r_{2} y_{2}}^{3}}^{3}+\boldsymbol{E}_{t_{r_{3} y_{3}}^{3}}^{3}+\ldots
$$

Ici $y_{1}, y_{2}, y_{3}, \ldots$, sont des variables indépendantes, donc les sommands sont indépendants l'un de l'autre; et si les

$$
E_{t_{r}}^{3}
$$

sont universels de certaines classes $P_{\beta_{n}}$ la somme donnera tous les ensembles (extraits de $J^{3}$ ) de la forme

$$
\sum P_{\beta_{n}}
$$

Cette somme

$$
E^{3}
$$

sera donc universelle pour la classe d'ordre $\max \beta_{n}$ ou $\lim \beta_{n}$. L'opération $P C P C$ augmentera ce nombre de 2 .

Pour l'interprétation de (1), remarquons ceci: si le paramètre $y$ parcourt ses valeurs, l'ensemble $\hat{x} \phi(0, x, y)$ parcourt toutes les sommes d'intervalles $(y, r)$ avec $y<r, y \in J$ et $r$ rationnel. Mais ces intervalles $(y, r)$ forment une base pour les ensembles ouverts dans $J$; donc $\hat{x} \phi(0, x, y)$ parcourt tous les ensembles ouverts, et $\hat{x} \hat{y} \phi(0, x, y)$ est un ensemble universel pour la classe $G$ des ensembles ouverts.

Ensuite, si ord $t=1$, l'ensemble $\hat{x} y \phi(t, x, y)$ est de la forme $P C P C \sum E^{3}$, où $E$ est universel pour la classe $G$, donc $\hat{x} \hat{y} \phi(t, x, y)$ sera universel pour la classe $P C P C G$, c'est à dire, pour la classe $P_{2}$ (puisque $P C P C G=P C P F=P C A$ $=P_{2}$, où $P F=A$, voir introduction).

De même, si ord $t=2$, l'ensemble $x \hat{y} \phi(t, x, y)$ sera universel pour la classe $P C P C \sum P_{2}=P_{4}$ (puisque la somme $\sum$, qui correspond à $(\exists r)$, est dénombrable), et ainsi de suite. Si ord $t=\omega$, on trouve $P C P C P_{\omega}=P_{\omega+2}$.

En général, si ord $t=\alpha$, l'ensemble $\hat{x} \hat{y} \phi(t, x, y)$ sera un ensemble universel pour une classe $P_{\beta}$, et on voit par induction transfinie que $\beta \rightarrow \Omega$ avec $\alpha \rightarrow \Omega$.

$$
\text { (Plus précisément, } \beta=\left\{\begin{array}{l}
2 n \text { pour } \alpha=n<\omega \\
2 \alpha+2 \text { pour } \alpha \geqslant \omega
\end{array}\right) .
$$

Nous pouvons donc, pour tout $\alpha$ effectif (c'est à dire, tel qu'on peut nommer un ensemble de nombres rationnels de ce type, ordonné rel $<$ ), nommer effectivement un ensemble universel de classe $P_{\alpha}$ au moins.

Notre construction montre aussi (voir introduction) l'existence d'ensembles proprement $P_{\alpha}$ pour tout $\alpha<\Omega$; et si $t, y$, parcourent toutes ses valeurs, l'ensemble $\hat{x} \phi(t, x, y)$ parcourt tous les ensembles projectifs de toutes les classes (finies ou transfinies). Ainsi, l'ensemble $\hat{t} \hat{x} \hat{y} \phi(t, x, y)$ (dans l'espace $J^{3}$ ) ne peut pas être projectif d'aucune classe: autrement, s'il était de classe 
$P_{\alpha_{0}}$, toutes les sections linéares $\hat{x} \phi(t, x, y)$ le seraient aussi, donc de classes bornées $\alpha \leqslant \alpha_{0}$. c.q.f.d. ${ }^{3}$

Toutefois, cette définition est, comme déjà dit, implicite (voir 2, p. 248) à cause de la définition (2). A présent, nous allons la rendre explicite moyennant un quantificateur du troisième ordre. Désignons par $\left(1^{\prime}\right),\left(2^{\prime}\right),\left(2^{* \prime}\right)$, les formules qu'on obtient de (1), $(2),\left(2^{*}\right)$, en remplacant $t, x, y$, respectivement par $t^{\prime}, x^{\prime}, y^{\prime}$. Voici la formule:

$$
\hat{t} \hat{x} \hat{y}(\exists \phi)\left(t^{\prime}\right)\left(x^{\prime}\right)\left(y^{\prime}\right):\left(1^{\prime}\right) . \& .\left(2^{* \prime}\right) \vee t^{\prime}=0 . \& . \phi(t, x, y)
$$

ou bien ce qui revient au même:

$$
\hat{t} \hat{x} \hat{y}(\phi):\left(t^{\prime}\right)\left(x^{\prime}\right)\left(y^{\prime}\right) .\left(1^{\prime}\right) . \& .\left(2^{* \prime}\right) \vee t^{\prime}=0 \rightarrow: \phi(t, x, y) .
$$

Ainsi, la définition explicite de cet ensemble est arithmétisable dans le calcul fonctionnel du troisième ordre, c.q.f.d.

5. Simplifications. Permettant des modifications assez légères et inessentielles de cet ensemble, on peut beaucoup simplifier sa définition.

D'abord, la définition initiale (1) peut être remplacée simplement par celleci:

$$
\phi(0, x, y) \cdot \equiv \cdot x<y<x+1 .
$$

Ensuite, sans changer les sections avec $t$ bien-ordonné dans l'ensemble (5), on peut remplacer $\left(2^{*}\right)$ par (2) et, comme nous allons voir, les équivalences par des implications, c'est à dire, remplacer (6) et (2) par

$$
\psi(0, x, y) \rightarrow x<y<x+1 \text { et } \psi(t, x, y) \rightarrow F \psi(t, x, y) \text { pour } t \neq 0,
$$

où $F \psi(t, x, y) \cdot \equiv \cdot(\exists z)\left(z^{\prime}\right)(\exists r) \psi\left(t_{r}, \pi_{3}\left(x, z, z^{\prime}\right), \pi_{r}(y)\right)$.

On obtient ainsi un autre ensemble non-projectif, mais tout à fait analogue, que voici:

(8) $\hat{t} \hat{x} \hat{y}(\exists \psi)$ (quant) $\left\{\psi\left(t^{\prime}, x^{\prime}, y^{\prime}\right): \rightarrow: A . \& . B \rightarrow \psi\left(t^{\prime \prime}, x^{\prime \prime}, y^{\prime \prime}\right)\right\} \& \psi(t, x, y)$,

$$
\text { où }\left\{\begin{array}{l}
\text { (quant })=\left(t^{\prime}\right)\left(x^{\prime}\right)\left(y^{\prime}\right)(\exists z)\left(z^{\prime}\right)(\exists r)\left(t^{\prime \prime}\right)\left(x^{\prime \prime}\right)\left(y^{\prime \prime}\right), \\
A \cdot \equiv \cdot t^{\prime} \neq 0 \vee x^{\prime}<y^{\prime}<x^{\prime}+1, \\
B \cdot \equiv \cdot t^{\prime} \neq 0 \& t^{\prime \prime}=t_{r}^{\prime} \& x^{\prime \prime}=\pi_{3}\left(x^{\prime} z, z^{\prime}\right) \& y^{\prime \prime}=\pi_{r}\left(y^{\prime}\right) .
\end{array}\right.
$$

Pour interpréter cette formule, il suffit de substituer respectivement $t^{\prime}=0$ et $t^{\prime} \neq 0$ dans $\{\ldots\}$; on trouvera précisément la condition (7) pour $\psi\left(t^{\prime}, x^{\prime}, y^{\prime}\right)$.

Reste à justifier le remplacement de “”” par “ $\rightarrow$ ”. Observons que, pour $t$ donné, $F \psi$ dépend seulement des $t_{r} \prec t$, et que $F$ est une "fonction croissante" en ce sens que

$$
\psi \rightarrow \phi: \rightarrow: F \psi \rightarrow F \phi \quad(t \neq 0)
$$

${ }^{3}$ Naturellement, cette non-projectivité peut aussi être démontrée sans difficultés sérieuses, par une adaptation du procédé de diagonale. 
Soit maintenant $\phi$ fixe satisfaisant à (6) et (2), donc $\phi \equiv F \phi$, et soit $\psi$ une fonction variable satisfaisant à

$$
\psi(0, x, y) \rightarrow \phi(0, x, y) \text { et } \psi \rightarrow F \psi \quad \text { pour } t \neq 0 .
$$

Considérons seulement les valeurs bien-ordonnées de $t$. On trouve par induction transfinie sur $t$ que

$$
\psi(t, x, y) \rightarrow \phi(t, x, y) \text {, donc ( } \exists \psi \text { satisf. à }(9)) \psi(t, x, y): \rightarrow \phi(t, x, y),
$$

d'où il vient sans difficulté

$$
\phi(t, x, y) \cdot \equiv \cdot(\exists \psi \text { satisf. à }(9)) \psi(t, x, y)
$$

pour $t$ bien-ordonné. Remarquant que (9) équivaut à (7), nous pouvons donc remplacer (6) et (2) par (7), ce qui nous donne facilement (8).

Cette formule (8) ne contient que trois occurences de $\psi$; mais on peut démontrer qu'une formule de cette sorte, avec une ou deux occurences seulement, donnerait toujours un ensemble projectif.

\section{BibLIOGRAPHIE}

1. C. Kuratowski, Topologie I (Warszawa-Lwów, 1933).

2. C. Kuratowski et A. Tarski, Les opérations logiques et les ensembles projectifs, Fund. Math., 17 (1931), 240-248.

3. N. Lusin, Leçons sur les ensembles analytiques (Paris, 1930).

4. W. Sierpiński, Les ensembles projectifs et analytiques (Mémorial des sciences mathématiques, Fasc. 112) (Paris, 1950).

5. - Sur les ensembles de points qu'on sait définir effectivement, Verhandlungen des internationalen Mathematiker-Kongresses Zürich, 1932, 1. Band, p. 280.

\section{Université Laval \\ Québec}

